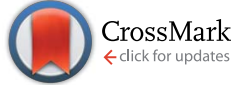

Cite this: Soft Matter, 2015, 11, 2423

Received 10th October 2014 Accepted 27th January 2015

DOI: $10.1039 / \mathrm{c} 4 \mathrm{sm} 02242 d$

www.rsc.org/softmatter

\section{Atomistic simulation for coil-to-globule transition of poly(2-dimethylaminoethyl methacrylate) $\uparrow$}

\begin{abstract}
Sa Hoon Min, ${ }^{a}$ Sang Kyu Kwak ${ }^{* b}$ and Byeong-Su Kim ${ }^{* a c}$
The coil-to-globule transition of poly(2-dimethylaminoethyl methacrylate) (PDMAEMA) in aqueous solution was investigated by all-atomistic molecular dynamics simulations. The polymer consistent force field (PCFF) was applied to the PDMAEMA model with a proper protonation state. The structural analysis indicates a distinct difference in the hydration state of particular functional groups of PDMAEMA as well as in the conformational state of PDMAEMA below and above the lower critical solution temperature (LCST). In particular, by monitoring the motion of water molecules, we observe that water molecules in the vicinity of the carbonyl group are relatively restricted to the motion in the globule state due to the extended relaxation time of hydrogen bonds among water molecules. The degree of protonation was also adjusted to study the effect of protonation on the conformational state of PDMAEMA.
\end{abstract}

\section{Introduction}

The transition between coil and globule states is a fundamental phenomenon in polymer chains. The conformational state of polymer chains in a good solvent exhibits a well expanded coil structure, while that in a poor solvent undergoes a collapse into a globule structure, due to the energetic difference in the interaction between the polymer and solvent molecules. ${ }^{\mathbf{1 1 2}}$ It has been observed that thermo-responsive polymers also show a similar conformational transition under the same solvent condition when changing the temperature through their lower critical solution temperature (LCST).$^{3-5}$ Among these polymers, poly( $N$-isopropylacrylamide) (PNIPAM), a representative thermo-responsive polymer, has been well studied by means of theoretical calculations ${ }^{6-9}$ as well as experimental methods ${ }^{10-12}$ to explore the conformational transition behaviour in an aqueous solution through the LCST whose range includes the human body temperature.

Recently, molecular dynamics (MD) simulations have been applied to observe what happens in the coil-to-globule transition of PNIPAM at the atomic level using diverse force-fields.

\footnotetext{
${ }^{a}$ Department of Energy Engineering, School of Energy and Chemical Engineering, Ulsan National Institute of Science and Technology (UNIST), Ulsan 689-798, Korea

${ }^{b}$ Department of Chemical Engineering, School of Energy and Chemical Engineering, Ulsan National Institute of Science and Technology (UNIST), Ulsan 689-798, Korea. E-mail: skkwak@unist.ac.kr; Tel: +82-52-217-2514

'Department of Chemistry, School of Natural Science, Ulsan National Institute of Science and Technology (UNIST), Ulsan 689-798, Korea. E-mail: bskim19@unist.ac. kr; Tel: $+82-52-217-2923$

$\uparrow$ Electronic supplementary information (ESI) available: The potential energy components of the PCFF, average temperatures of PDMAEMA and water molecules, radius of gyration for replica simulations, intramolecular pairwise energy of PDMAEMA in the extension of the production run, and total system energies. See DOI: $10.1039 / \mathrm{c} 4 \mathrm{sm} 02242 \mathrm{~d}$
}

The optimized-potentials-for-liquid-simulations (OPLS) forcefield has been successfully used by Spohr's group to investigate the LCST of multiple chains as well as a single chain of PNIPAM in water, showing that the polar amide group could be a temperature-sensitive region for the coil-to-globule transition. ${ }^{\mathbf{1 3}}$ In addition, they have modelled a PNIPAM-grafted cylindrical pore $^{\mathbf{1 4}}$ and graphene-like nanosheets connected with PNIPAM ${ }^{15}$ to predict the temperature-responsive properties on the inner surface functionalized pore and on the conceptual nanoengines, respectively, by the assisted model building with the energy refinement (AMBER) 94 force-field. In other examples, with this force-field, Longhi et al. reported the microscopic details between a 50-mer of PNIPAM and water molecules below and above the LCST, ${ }^{16}$ and Du et al. also reported the effect of salt on the LCST of PNIPAM by calculating the radius of gyration $\left(R_{\mathrm{g}}\right)$ and the radial distribution function (RDF) between atoms in PNIPAM and the salt cations. ${ }^{17}$ The DREIDING force-field was applied to study the PNIPAM-grafted silicon substrate for understanding the deswelling process above the LCST. ${ }^{\mathbf{1 8}}$

In particular, Mancini's group have mainly focused on the polymer consistent force field (PCFF) to simulate the coil-toglobule transition of a 30-mer of PNIPAM in water. ${ }^{19-21}$ They observed a polymer chain length dependence on the conformational transition, indicating that short PNIPAM oligomers such as 3-, 5-, and 10-mer did not change their conformation significantly. Moreover, their studies revealed that there was a distinct difference in the hydrogen bond (H-bond) strength between PNIPAM-water and water-water below and above the LCST by analysing the $\mathrm{H}$-bond autocorrelation functions and the vibrational spectra of the water molecules. ${ }^{\mathbf{2 0 2 1}}$ These simulation results are in good agreement with the experimental observation, and give an insight into the structural and dynamic correlations between the polymer and water molecules below 
and above the LCST. Even though it could not elucidate the exact mechanism of the coil-to-globule transition, it would help to understand a possible driving force for the transition.

Poly(2-dimethylaminoethyl methacrylate) (PDMAEMA), a weak cationic polyelectrolyte, has been widely used as non-viral gene delivery vectors with buffering capacity and low cytotoxicity. ${ }^{22-26}$ In contrast with PNIPAM, PDMAEMA is another class of thermo-responsive polymer whose phase transition is also affected by the $\mathrm{pH}$ of solutions, resulting from the protonationdeprotonation of the tertiary amine group in PDMAEMA. ${ }^{27-31}$ Copolymerization with the DMAEMA monomer makes it possible to design multi-responsive polymeric micelles where PDMAEMA plays a crucial role in thermo- and $\mathrm{pH}$-responsiveness, simultaneously. ${ }^{32-37}$ It has been considered that this can be applied in the smart drug delivery system. ${ }^{38,39}$ Furthermore, PDMAEMA has provided the opportunities for the functionalization of the dual responsiveness on nanomaterials such as nanoparticles, ${ }^{40,41}$ nanoclay ${ }^{42}$ fullerene, ${ }^{43}$ and graphene. ${ }^{44}$

Owing to the protonation of amine groups, the conformational transition of PDMAEMA in aqueous solution is a more complicated phenomenon than the PNIPAM case. Thus, it is still challenging to study its coil-to-globule transition, causing the thermo- and pH-responsive properties. Plamper's group recently reported the role of the carbonyl group and polymer backbone in the conformational transition with fluorescence spectroscopy. ${ }^{45}$ However, the coil-to-globule transition of PDMAEMA with temperature changes has not been studied yet by the all-atomistic MD simulation, which can provide insights into the origin of the conformational transition, despite numerous theoretical studies on PNIPAM.

In this study, we perform MD simulations for the first time to investigate the coil-to-globule transition of PDMAEMA in the aqueous phase through a model system established for a 30mer of PDMAEMA in water with a proper protonation state. The MD simulations were employed to observe the coil-to-globule transition of PDMAEMA at three different temperature regimes. The structural correlations between PDMAEMA and water molecules were analysed for both the coil and globule states. In particular, we monitored the motion of water molecules in the vicinity of the specific functional groups in PDMAEMA to calculate the dynamic properties of water, such as the residence time and the $\mathrm{H}$-bond relaxation time around PDMAEMA to account for the origin of the coil-to-globule transition in PDMAEMA. The degree of protonation of PDMAEMA was also adjusted to investigate the effect of protonation on the conformational state.

\section{Method}

\section{Force-field and polymer modelling}

For the all-atomistic model of PDMAEMA, the PCFF ${ }^{\mathbf{4 6 - 4 8}}$ as an $a b$ initio based class II force-field was chosen because it has been successfully applied in the coil-to-globule transition of polymers ${ }^{19-21}$ as well as the prediction for the physical properties of polymers. ${ }^{49,50}$ The potential energy of the system can be calculated as a summation of valence interactions, valence crossterms, and non-bonding interactions by the following eqn (1).
Detailed energy expressions for each potential are described in the ESI. $\dagger$

$$
\begin{aligned}
E_{\text {total }}= & E_{\text {bond }}+E_{\text {angle }}+E_{\text {torsion }}+E_{\text {oop }}+E_{\text {cross-term }} \\
& +E_{\text {Coulomb }}+E_{\mathrm{vdW}}
\end{aligned}
$$

In the case of a short polymer chain, it is considered that there is a limitation in conformational transition of polymers. ${ }^{19,51}$ Thus, we modelled a single PDMAEMA chain comprised of 30 monomer units, which is long enough to form both the coil and globule structure depending on temperature changes. ${ }^{51}$ In addition, the atactic form was applied to the PDMAEMA model to reproduce the general PDMAEMA chain. ${ }^{52,53}$ The protonation state of PDMAEMA varies with the $\mathrm{pH}$ of the solution due to the protonation-deprotonation of the nitrogen atom in the dimethylamino (DMA) group. Recent experimental studies suggest the $\mathrm{pH}$-dependent protonation properties, indicating that PDMAEMA is completely protonated at a low $\mathrm{pH}$ level and can be deprotonated with increasing $\mathrm{pH}$ of the solution..$^{29,52}$ In particular, the degree of protonation of PDMAEMA is known to be about 0.5 at neutral $\mathrm{pH}$, owing to the $\mathrm{p} K_{\mathrm{a}}$ of 7.0-7.3 for PDMAEMA. ${ }^{29}$ To simulate the coil-to-globule transition of PDMAEMA in neutral water, these protonation states were employed in a 30-mer of PDMAEMA model by an alternate protonation, as shown in Fig. 1. The degree of protonation was further adjusted to achieve pH-dependent transition behaviour of PDMAEMA.

\section{Simulation details}

All of the MD simulation studies were performed with the LAMMPS code (http://lammps.sandia.gov) ${ }^{54}$ and the GPUaccelerated package. ${ }^{55}$ The PCFF was used for both PDMAEMA and water molecules ${ }^{\mathbf{1 9 , 5 6 , 5 7}}$ in a cubic simulation box with the periodic boundary conditions for all directions. The simulations were calculated with the isothermal-isobaric (NPT) ensemble, and the target temperature and pressure were maintained by using the Nose-Hoover thermostat and barostat with damping relaxation times of 1 and $2 \mathrm{ps}$, respectively. A cutoff of 9.5 Å was applied to calculate the pairwise interactions, and the long-range electrostatic interactions were evaluated using the particle-particle particle-mesh (PPPM) method with an RMS accuracy of $10^{-4}$. The bond lengths and angles for the
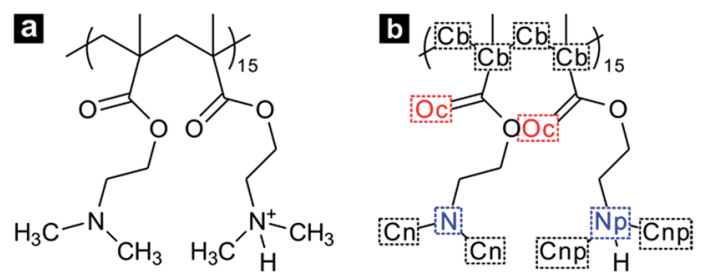

Fig. 1 (a) Structure of a PDMAEMA 30-mer with alternate protonation states and (b) the notation for specific atoms, such as carbon atoms in the PDMAEMA backbone ( $\mathrm{Cb})$, oxygen atoms in the carbonyl group $(\mathrm{Oc})$, nitrogen $(\mathrm{N})$ and carbon $(\mathrm{Cn})$ atoms in the unprotonated dimethylamino (DMA) group, and nitrogen ( $\mathrm{Np}$ ) and carbon ( $\mathrm{Cnp}$ ) atoms in the protonated DMA group. 
molecules were not constrained, thus a time step of $1 \mathrm{fs}$ with a velocity-Verlet scheme was employed for the integration of motion.

A single PDMAEMA chain with a fully extended backbone was placed in a simulation box with 9000 water molecules, resulting in the initial density of about $1.0 \mathrm{~g} \mathrm{~cm}^{-3}$. For the protonated PDMAEMA, appropriate counterions $\left(\mathrm{Cl}^{-}\right)$were added to neutralize the simulation system. The initial topologies and force-field parameters were built by Materials Studio®, and were converted into the LAMMPS-readable format. After energy minimization for unfavourable geometry of initial topologies, short simulations consisting of a 100 ps canonical (NVT) ensemble followed by a 900 ps NPT ensemble under atmospheric pressure were carried out at $283 \mathrm{~K}$ for the initial equilibrium of the system. Long NPT simulations of $30 \mathrm{~ns}$ for the production runs were performed at three different temperatures of 283,303 , and $338 \mathrm{~K}$ in order to investigate the coil-toglobule transition depending on temperature. The structural and dynamical analyses were performed using the VMD package. $^{58}$

\section{Results and discussion}

\section{Structural analysis}

In aqueous solution, PDMAEMA exhibits a phase separation behaviour induced by the conformational transition with an LCST around $40{ }^{\circ} \mathrm{C}$, which can be affected by the molecular weight, solution $\mathrm{pH}$, and concentration. ${ }^{30}$ Thus, the MD simulations were carried out at 283 and $303 \mathrm{~K}$, i.e. below the LCST, and at $338 \mathrm{~K}$, i.e. above the LCST of PDMAEMA. During the simulation, the system temperature was well maintained by the Nose-Hoover thermostat (Fig. S1 $\dagger$ ). Fig. 2a shows the time evolution of the radius of gyration $\left(R_{\mathrm{g}}\right)$ for PDMAEMA at three different temperature conditions. $R_{\mathrm{g}}$ which represents the dimension of a polymer chain is calculated by averaging the mass-weighted distance of each atom from the center-of-mass position of the group of atoms:

$$
R_{\mathrm{g}}{ }^{2}=\frac{1}{M} \sum_{i} m_{i}\left(r_{i}-r_{\mathrm{cm}}\right)^{2},
$$

where $M$ is the total mass of the group, $m_{i}$ is the mass of atom $i$, $r_{i}$ is the position of atom $i$, and $r_{\mathrm{cm}}$ is the center-of-mass position of the group. After sharp fluctuations at the early stage, the $R_{\mathrm{g}}$ of PDMAEMA at both 283 and $303 \mathrm{~K}$ reached a steady state with an averaged value of 16.3 and $15.7 \AA$ for the last $10 \mathrm{~ns}$, respectively. In contrast, the $R_{\mathrm{g}}$ of PDMAEMA above the LSCT decreased sharply over the time trajectory, and reached a value of $10.8 \AA$ by averaging the last $10 \mathrm{~ns}$ of individual trajectories. This indicates a distinct transition of conformation for PDMAEMA compared with the $R_{\mathrm{g}}$ below the LCST. The conformational transitions of PDMAEMA were also confirmed by a different initial state (Fig. S2†). Since the polymer conformation could also affect solvent accessibility to the polymer chain, the water accessible surface area (WASA) was calculated by rolling a water probe of radius $1.4 \AA$ across the van der Waals surface of PDMAEMA with the Shrake-Rupley algorithm ${ }^{59}$ in the VMD. The time evolution of WASA shows the similar tendency to that of $R_{\mathrm{g}}$ as shown in
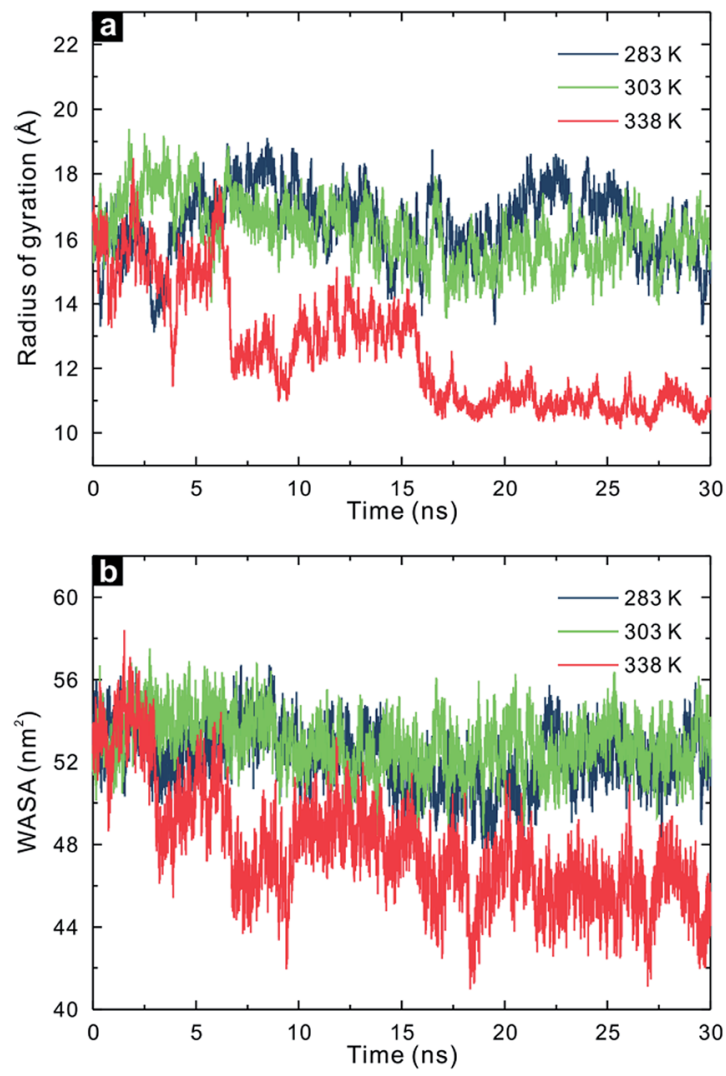

Fig. 2 Time evolution of (a) the radius of gyration, $R_{\mathrm{g}}$, and (b) the water accessible surface area, WASA, for PDMAEMA at $283 \mathrm{~K}$ (blue), $303 \mathrm{~K}$ (green), and $338 \mathrm{~K}$ (red).

Fig. 2b. In particular, the WASA values were found to be 52.0 and $52.7 \mathrm{~nm}^{2}$ in the case of 283 and $303 \mathrm{~K}$, while that decreased to $45.9 \mathrm{~nm}^{2}$ at $338 \mathrm{~K}$. The conformations of PDMAEMA below the LCST maintain the accessibility of water molecules, whereas those above the LCST lose the accessibility, which leads to the decrease in the surface area.

Indeed, the equilibrium morphologies of PDMAEMA are consistent with each $R_{\mathrm{g}}$ and WASA as shown in Fig. 3. After the production runs for $30 \mathrm{~ns}$, coil states of PDMAEMA with a partially linear structure are observed at 283 and $303 \mathrm{~K}$, and a globule state with a compact configuration is observed at $338 \mathrm{~K}$. These structural properties represent a distinct difference in the domain size of PDMAEMA through the LCST. From the atomic viewpoint, we further focused on the structural features between PDMAEMA and water molecules in the coil and globule states.

The radial distribution function (RDF) makes it possible to calculate the probability of finding atom $i$ at a distance $r$ from atom $j$, meaning the local structure between atoms $i$ and $j$. The RDF can be defined by eqn (3),

$$
g_{j i}(r)=\left(\frac{n_{i}}{4 \pi r^{2} \mathrm{~d} r}\right) /\left(\frac{N_{i}}{V}\right)
$$

where $n_{i}$ is the number of atoms $i$ in a shell thickness $\mathrm{d} r$ from atom $j, N_{i}$ is the total number of atoms $i$, and $V$ is the volume of the system. To understand the microstructures of the coil and 
a
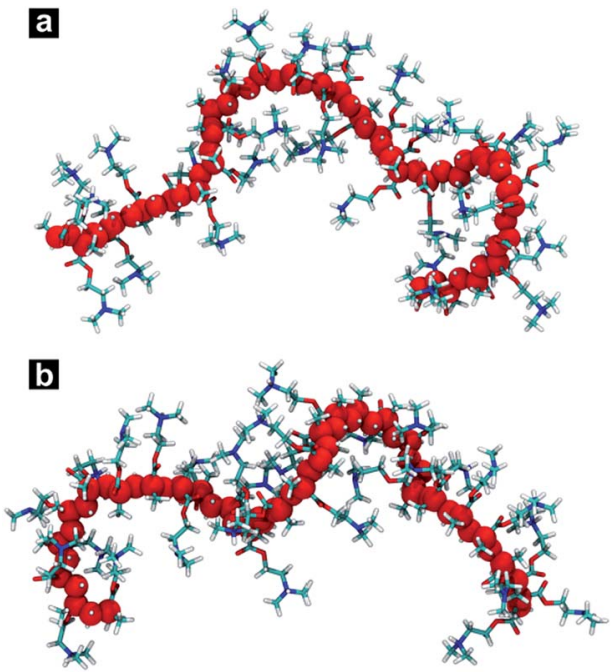

c

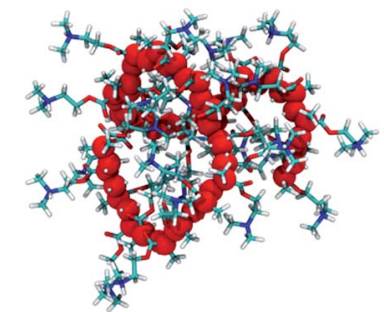

Fig. 3 Equilibrium morphologies of PDMAEMA from the last trajectory of the 30 ns simulation at (a) $283 \mathrm{~K}$, (b) $303 \mathrm{~K}$, and (c) $338 \mathrm{~K}$. Water molecules are not shown, and carbon atoms in the backbone are highlighted with large red beads, for clarity.

globule state of PDMAEMA with water molecules, the RDFs of the specific atoms in the functional groups of PDMAEMA to oxygen in water were calculated and averaged for the last $10 \mathrm{~ns}$ of each long NPT simulation.

Fig. 4a and b display the RDFs of the carbon (Cn) and nitrogen $(\mathrm{N})$ atom in the unprotonated DMA group to the oxygen (Ow) atom in water, respectively. While there were no significant differences in the intensity between the RDF peak at 283 and $303 \mathrm{~K}$, the RDFs at $338 \mathrm{~K}$ showed a considerable decrease in the intensity compared with that below the LCST. By integrating the RDFs, the number of water molecules $\left(N_{\mathrm{w}}\right)$ was calculated for the first and second hydration shells surrounding the two different DMA groups of PDMAEMA, as presented in Table 1 . The $N_{\mathrm{w}}$ in the second hydration shell as well as the first hydration shell of $\mathrm{Cn}$ and $\mathrm{N}$ was significantly reduced by $>35 \%$ at $338 \mathrm{~K}$. It indicates that the unprotonated DMA groups of PDMAEMA tend to be shielded from water molecules in its globule state, since the probability of finding water molecules remarkably decreases from $\mathrm{Cn}$ and $\mathrm{N}$ atoms above the LCST.

Table 1 Average number of water molecules $\left(N_{w}\right)$ in the first and second hydration shells of the specific atoms in the unprotonated DMA, the protonated DMA, and the carbonyl groups ${ }^{a}$

\begin{tabular}{llll}
\hline & & & \\
\cline { 3 - 4 } & & & \\
& & & \\
Temperature (K) & First shell $^{b}$ & Second shell $^{c}$ \\
& 283 & $8.47 \pm 0.28$ & $30.49 \pm 0.74$ \\
& 303 & $8.11 \pm 0.23$ & $29.33 \pm 0.56$ \\
$\mathrm{~N}$ & 338 & $5.18 \pm 0.31$ & $19.83 \pm 1.01$ \\
& 283 & $0.36 \pm 0.03$ & $17.26 \pm 0.49$ \\
& 303 & $0.33 \pm 0.02$ & $16.73 \pm 0.34$ \\
$\mathrm{Cnp}$ & 338 & $0.20 \pm 0.02$ & $11.05 \pm 0.57$ \\
& 283 & $9.15 \pm 0.07$ & $32.93 \pm 0.16$ \\
$\mathrm{~Np}$ & 303 & $9.09 \pm 0.06$ & $33.12 \pm 0.20$ \\
& 338 & $8.68 \pm 0.07$ & $31.94 \pm 0.20$ \\
& 283 & $1.06 \pm 0.01$ & $21.91 \pm 0.12$ \\
Oc & 303 & $1.04 \pm 0.01$ & $22.06 \pm 0.16$ \\
& 338 & $1.02 \pm 0.01$ & $21.43 \pm 0.16$ \\
& 283 & $1.98 \pm 0.02$ & \\
& 303 & $1.88 \pm 0.04$ & \\
& 338 & $1.53 \pm 0.04$ &
\end{tabular}

${ }^{a}$ The $N_{\mathrm{w}}$ is calculated from the last $10 \mathrm{~ns}$ trajectory. ${ }^{b}$ Integration from zero to the first minimum of each RDF. ${ }^{c}$ Integration from the first minimum to the second minimum of each RDF.
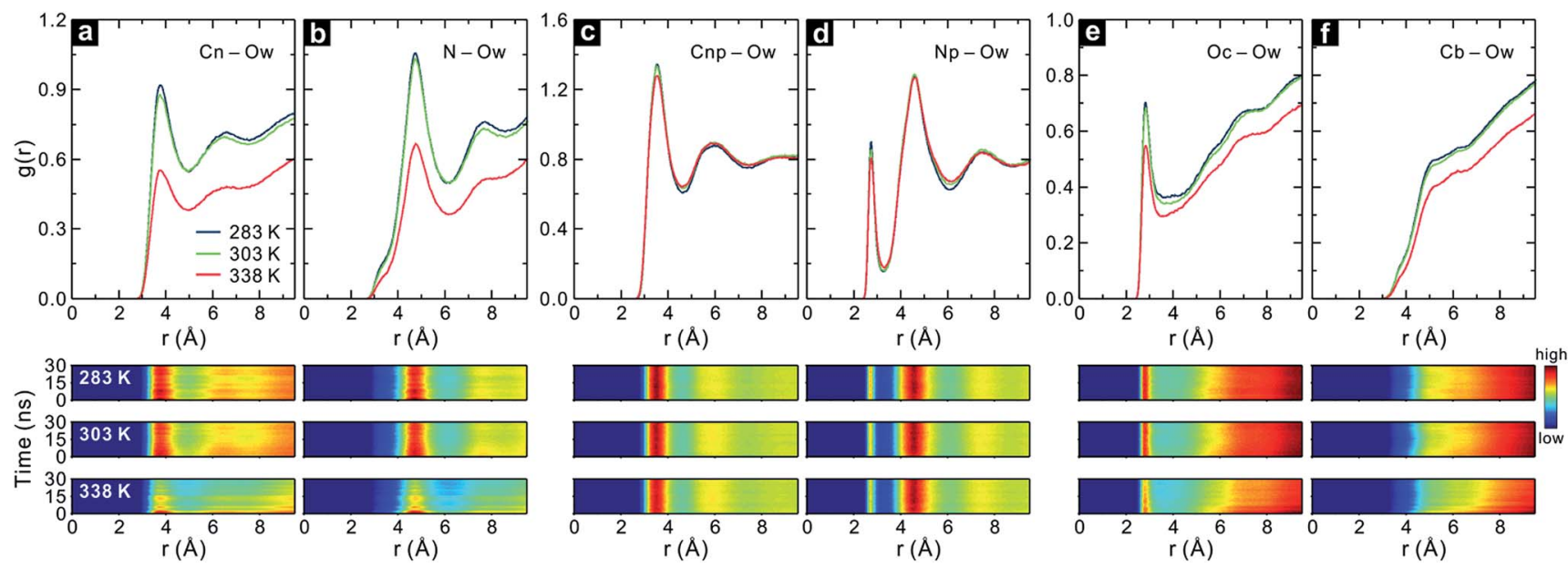

Fig. 4 Radial distribution functions (RDFs) of (a) $\mathrm{Cn}$ and (b) N in the unprotonated DMA group, (c) Cnp and (d) Np in the protonated DMA group, (e) Oc in the carbonyl group, and (f) $\mathrm{Cb}$ in the backbone to water oxygen (OW) at 283, 303, and $338 \mathrm{~K}$. The corresponding contour plots of 1 ns averaged RDF are shown at the bottom of each graph. 
In Fig. 4c, it can be seen that the intensities of the RDFs between the carbon (Cnp) atom in the protonated DMA group and $\mathrm{Ow}$ increase in comparison with that between $\mathrm{Cn}$ and $\mathrm{Ow}$ (Fig. 4a), and the $N_{\mathrm{w}}$ in the first and second hydration shell is also found to be higher for all temperature ranges. In addition, the peak positions of the RDFs are slightly shifted toward smaller distance by $0.3 \AA$. It suggests that the methyl groups in the protonated DMA group have a stronger interaction with water molecules than those in the unprotonated DMA group, due to the change of partial charge by the protonation. Fig. $4 \mathrm{~d}$ also represents an increase in the intensities of the RDFs between the nitrogen ( $\mathrm{Np}$ ) in the protonated DMA group and Ow, compared with Fig. 4b. In particular, sharp peaks located at $2.7 \AA$ A were observed, resulting from the $\mathrm{H}$-bond between $\mathrm{Np}$ and Ow atoms, while there were only weak shoulder peaks in the $\mathrm{N}-\mathrm{Ow}$ RDFs at the same position. It also reveals a distinct difference between the number of water molecules in the first hydration shell of $\mathrm{N}$ and $\mathrm{Np}$, which can be related to water molecules participating in the $\mathrm{H}$-bond to each DMA group. Interestingly, in the case of the protonated DMA group, the RDFs above the LCST are almost similar to those below the LCST. The intensities of the RDFs do not decrease much even in its globule state, suggesting that the structural correlation between the protonated DMA group and water molecules remains stable regardless of the conformational states. In other words, the protonated DMA groups are not shielded from water molecules even in the globule state. The $N_{\mathrm{w}}$ also exhibits a slight decrease $(<5 \%)$ for the first and second hydration shells of Cnp and $\mathrm{Np}$ at $338 \mathrm{~K}$. Moreover, the contour plots of the protonated DMA group show the steady state values of RDF over the simulation time in contrast with the other functional groups. It supports the fact that the protonated DMA groups are not consistently shielded from water molecules during the coil-toglobule transition.

Fig. 4e and $\mathrm{f}$ exhibit the RDFs between the carbonyl oxygen (Oc) and Ow, and between the backbone carbon $(\mathrm{Cb})$ and Ow, respectively. As with the unprotonated DMA group, the intensity of the RDFs for the carbonyl group and polymer backbone decreased above the LCST. Due to the absence of a clear second minimum of the Oc-Ow RDFs, we only calculated the $N_{\mathrm{w}}$ in the first hydration shell of Oc which decreased by $23 \%$ at $338 \mathrm{~K}$, compared with that at $283 \mathrm{~K}$. This expulsion of water in the vicinity of the carbonyl group and polymer backbone during the course of coil-to-globule transition has also been similarly observed in MD simulations of PNIPAM. ${ }^{\mathbf{1 4 , 1 8 , 1 9}}$

Overall, judging from the RDFs, we observe that the structural correlation between the DMA groups and water is affected by the protonation. Each DMA group in the coil state of PDMAEMA maintains the microstructure with water molecules. However, in the globule state (i.e., above the LCST), the unprotonated DMA groups are mainly shielded inside the globule state, while the protonated DMA groups are exposed on the surface of the globule state. Moreover, the carbonyl group and backbone of PDMAEMA also lose their contact with water molecules in the globule state.

This interesting conformational transition of PDMAEMA was also revealed by the pairwise energy during the production run.
Fig. 5a shows the time averaged intramolecular pairwise energies of PDMAEMA. The coil states of PDMAEMA mostly maintained its self-interaction energy, indicating that there was not an appreciable change in the intramolecular structure. However, owing to the conformational change, the self-interaction energy gradually increased through the globule transition and was well converged as shown in the extension of the simulation (Fig. S3†). In the case of the intermolecular pairwise energy between PDMAEMA and water, the pairwise energy decreases with increasing temperature, as shown in Fig. $5 \mathrm{~b}$. The pairwise energy of the coil state was further reduced compared to the thermal effect. This decrease in the interaction energy between PDMAEMA and water molecules also supports the fact that the functional groups in PDMAEMA are shielded from water molecules above the LCST.

\section{H-bond analysis}

In the structural analysis of the coil and globule state of PDMAEMA, there is a difference not only in the dimension of PDMAEMA but also in the microstructure of water molecules in the vicinity of the functional groups. Since it has been discussed that the H-bond plays an important role in the coil-to-globule transition of a single chain of PNIPAM, ${ }^{\mathbf{1 6}, 19}$ we focused on the unprotonated DMA, protonated DMA, and carbonyl group, which can participate in the formation of $\mathrm{H}$-bonding. The $\mathrm{H}$ bond in this study was defined by using the geometric criteria of the donor and acceptor. Among the atoms in the candidate group for the $\mathrm{H}$-bond, a pair can be considered to participate in $\mathrm{H}$-bond formation if the distance between the donor and acceptor is less than $3.6 \AA$, and the angle between hydrogendonor and donor-acceptor is less than $30^{\circ}$, simultaneously. The number of $\mathrm{H}$-bonds was averaged for the last $10 \mathrm{~ns}$ of the production run.

Fig. 6 shows the number of $\mathrm{H}$-bonds per each functional group between the specific functional groups (i.e., the unprotonated DMA, the protonated DMA, and the carbonyl group) and water molecules, respectively. It can be seen that there are few H-bonds between the unprotonated DMA group and water.
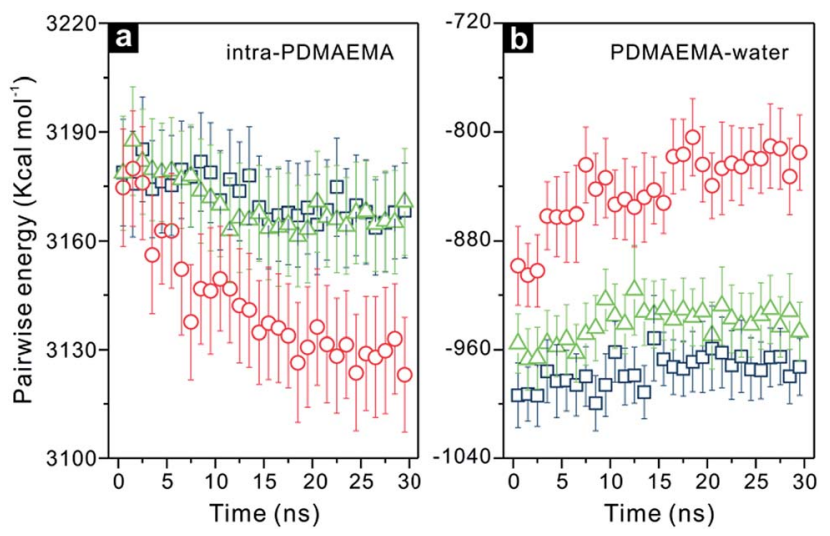

Fig. 5 Time series of (a) the intramolecular pairwise energies of PDMAEMA and (b) the intermolecular pairwise energies between PDMAEMA and water at 283, 303, and $338 \mathrm{~K}$. 


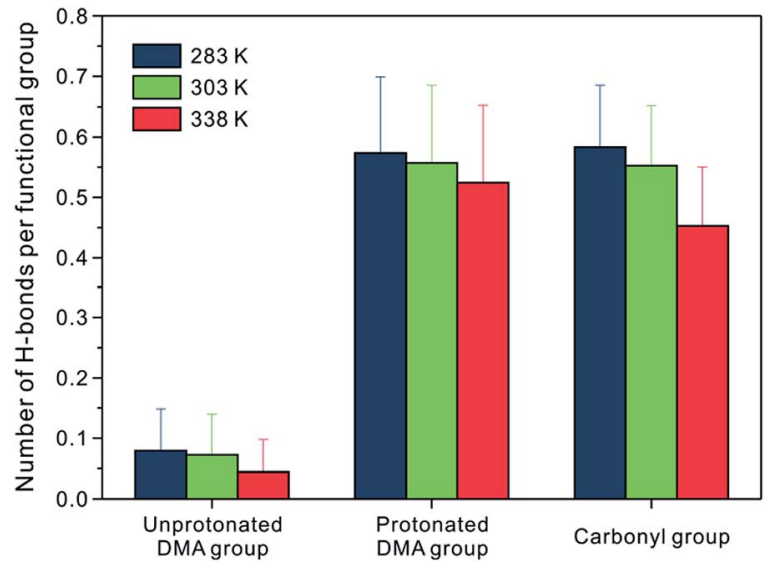

Fig. 6 Average number of $\mathrm{H}$-bonds per each functional group unit between $\mathrm{N}$ and $\mathrm{OW}$ in the unprotonated DMA group, between Np and Ow in the protonated DMA group, and between Oc and Ow in the carbonyl group at 283, 303, and $338 \mathrm{~K}$.

At $283 \mathrm{~K}$, only $8 \%$ of the unprotonated DMA groups form an $\mathrm{H}$ bond with water molecules, suggesting that the unprotonated DMA group is a poor $\mathrm{H}$-acceptor for $\mathrm{H}$-bond. However, owing to the polar nature of the protonated DMA group and the carbonyl group, $57 \%$ of $\mathrm{Np}$ and $58 \%$ of Oc atoms have $\mathrm{H}$-bonds with water molecules. At $303 \mathrm{~K}$, which is still in a coil state, the decrease in the number of $\mathrm{H}$-bonds is insignificant for all functional groups. It is noteworthy that the number of H-bonds between the protonated DMA group and water molecules almost remains the same in the globule state. It indicates that the protonated DMA group maintains the H-bonds to water as well as the microstructure with water molecules regardless of the conformational state. On the other hand, the number of $\mathrm{H}$ bonds for $\mathrm{N}-\mathrm{Ow}$ and Oc-Ow pairs decreases by $44 \%$ and $22 \%$ in the globule state, respectively. These decreases in the number of $\mathrm{H}$-bonds well correlate with that in the $N_{\mathrm{w}}$ for the first hydration shell of the N-Ow and Oc-Ow RDFs, as shown in Fig. 4, which show a $43 \%$ and $23 \%$ reduction in the $N_{\mathrm{w}}$ at $338 \mathrm{~K}$, respectively. Thus, the water molecules in the first hydration shell particularly involved in $\mathrm{H}$-bonds to each functional group. ${ }^{16}$ Note that the unprotonated DMA group hardly participates in $\mathrm{H}$-bond to water molecules. The difference between the total number of $\mathrm{H}$ bonds for the $\mathrm{N}-\mathrm{Ow}$ pair at 283 and $338 \mathrm{~K}$ is as little as 0.5 , whereas that for the Oc-Ow pair at 283 and $338 \mathrm{~K}$ is about 3.9. Therefore, we can conclude that the carbonyl group in PDMAEMA shows a considerable decrease in the number of $\mathrm{H}$ bonds through the coil-to-globule transition.

Previous studies on PNIPAM have shown that the intrachain $\mathrm{H}$-bonds between the amide nitrogen and the carbonyl oxygen in PNIPAM exist in its globule state, and can play a crucial role in the interaction for the coil-to-globule transition. ${ }^{\mathbf{1 3 , 2 0}}$ In a stark contrast, we found interestingly that the intrachain $\mathrm{H}$-bonds in PDMAEMA rarely existed $(<0.1 \%)$ in its globule state. Although the atomistic simulation in this study is limited to the polymer length of 30-mer, which can lead to enhanced steric hindrance for the intramolecular $\mathrm{H}$-bond, it suggests that the intrachain $\mathrm{H}$-bond between the protonated DMA group and the carbonyl group is not a dominant factor for the coil-to-globule transition of PDMAEMA.

To investigate more detailed motion of water molecules surrounding the unprotonated DMA, protonated DMA, and carbonyl group, additional NPT simulations of 200 ps were sequentially performed five times from the last trajectories at each temperature, with the time interval of 0.01 ps for sampling. The $\mathrm{H}$-bond relaxation time can be calculated for the $\mathrm{H}$-bond not only between PDMAEMA and water but also among water molecules in the vicinity of the functional groups of PDMAEMA from the normalized time correlation function (TCF) as shown below,

$$
c_{\mathrm{x}}(t)=\left\langle h_{i j}(t) h_{i j}\left(t_{0}\right)\right\rangle /\left\langle h_{i j}\left(t_{0}\right) h_{i j}\left(t_{0}\right)\right\rangle
$$

where $h_{i j}(t)=1$ if atoms $i$ and $j$ are hydrogen-bonded from time $t_{0}$ to $t$, and it is zero otherwise. $\mathrm{x}$ is pw or ww for the case of the $\mathrm{H}$-bond between PDMAEMA and water, and among water molecules in the vicinity of the specific functional group, respectively. $h_{i j}(t)$ is not affected when breaking or reforming $\mathrm{H}$ bonds at an intermediate time, thus it is compliant with the intermittent $\mathrm{H}$-bond $\mathrm{TCF}^{60}$ The angular brackets denote an average over all pairs. In the case of the H-bond among water molecules in the vicinity of each functional group, water molecules which are within $6.1 \AA$ of the N, Np, and Oc atom are only monitored for the unprotonated DMA, the protonated DMA, and the carbonyl group, respectively. These regions are equal up to the second hydration shell of the DMA groups. Since there is no distinct second minimum of Oc-Ow RDFs, a region of the same size is used for the carbonyl group. The H-bond TCFs are fitted to obtain the mean $\mathrm{H}$-bond relaxation time by the multi-exponential function, as follows,

$$
\begin{gathered}
c_{\mathrm{x}}(t)=\sum_{i=1}^{n} c_{i} \exp \left(-\frac{t}{\tau_{i}}\right) \\
\tau_{\mathrm{x}}=\sum_{i=1}^{n} c_{i} \tau_{i}
\end{gathered}
$$

where the sum of $c_{i}$ is unity, and $n=3$ or 4 for the case of the $\mathrm{H}$ bond between PDMAEMA and water, and among water molecules, respectively. The mean relaxation time of $\mathrm{H}$-bonds $\tau_{\mathrm{x}}$ is calculated by summing the $c_{i} \tau_{i}$.

Fig. 7a-c exhibit the relaxation behaviour of the H-bond between PDMAEMA and water. It is observed that the relaxation of H-bonds decays faster with increasing temperature due to the thermal energy. Among the three different functional groups, the $c_{\mathrm{pw}}(t)$ of the unprotonated DMA group shows the fastest decay with the mean relaxation time of the $\mathrm{H}$-bond between PDMAEMA and water $\left(\tau_{\mathrm{pw}}\right)$ below 1 ps at all temperature conditions, as summarized in Table 2 . It suggests that the $\mathrm{H}$ bond between the unprotonated DMA nitrogen and water oxygen has poor stability. The relaxation behaviour of the $c_{\mathrm{pw}}(t)$ of the protonated DMA group and the carbonyl group is much slower than that of the unprotonated DMA group, indicating that the functional groups maintain stable $\mathrm{H}$-bonds to water molecules. The $\tau_{\mathrm{pw}}$ of the protonated DMA group is always larger than that of the carbonyl group, but the difference 

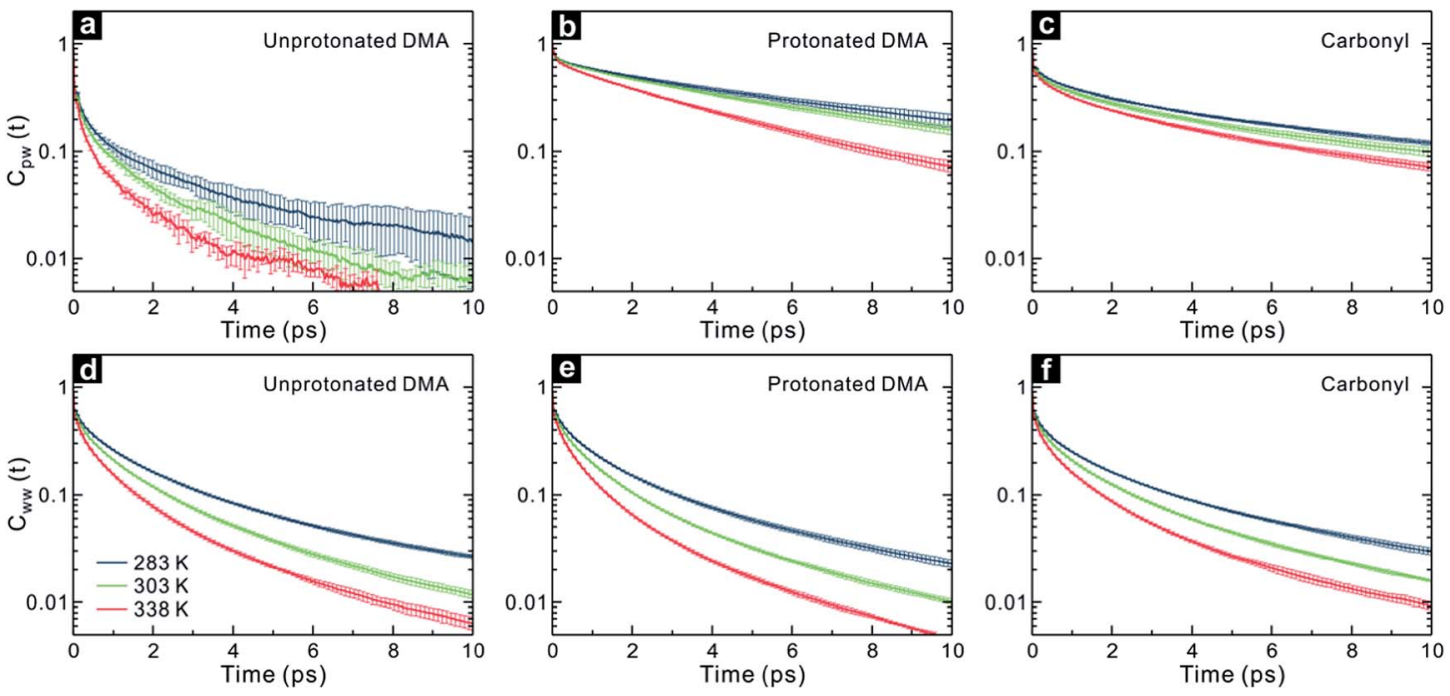

Fig. 7 Semi-log plots of time correlation function (TCF) of the $\mathrm{H}$-bond between (a-c) PDMAEMA and water, and between ( $d-f$ ) water molecules in the vicinity of the specific functional groups at 283,303 and $338 \mathrm{~K}$.

Table 2 Mean relaxation time of $\mathrm{H}$-bonds for PDMAEMA-water and water-water pairs in the vicinity of PDMAEMA

\begin{tabular}{llll}
\hline & & \multicolumn{2}{l}{ H-bond relaxation time ${ }^{a}(\mathrm{ps})$} \\
\cline { 3 - 4 } Functional group & Temperature $(\mathrm{K})$ & $\tau_{\mathrm{pw}}{ }^{b}$ & $\tau_{\mathrm{ww}}{ }^{b}$ \\
\hline Unprotonated DMA & 283 & $0.76 \pm 0.38$ & $1.38 \pm 0.06$ \\
& 303 & $0.38 \pm 0.06$ & $0.88 \pm 0.02$ \\
Protonated DMA & 338 & $0.24 \pm 0.03$ & $0.61 \pm 0.01$ \\
& 283 & $6.39 \pm 0.94$ & $1.23 \pm 0.05$ \\
Carbonyl & 303 & $5.40 \pm 0.71$ & $0.81 \pm 0.01$ \\
& 338 & $3.04 \pm 0.26$ & $0.54 \pm 0.01$ \\
& 283 & $4.27 \pm 0.36$ & $1.37 \pm 0.04$ \\
& 303 & $3.55 \pm 0.31$ & $0.96 \pm 0.02$ \\
& 338 & $2.70 \pm 0.48$ & $0.68 \pm 0.02$
\end{tabular}

${ }^{a}$ The mean H-bond relaxation time is calculated by multi-exponential fitting the H-bond TCF. ${ }^{b} \tau_{\mathrm{pw}}$ and $\tau_{\mathrm{ww}}$ are the mean relaxation time of the H-bond between PDMAEMA and water, and among water molecules in the vicinity of each functional group, respectively.

diminishes with increasing temperature, for example, at $338 \mathrm{~K}$, the difference is only 0.3 ps.

Fig. $7 \mathrm{~d}-\mathrm{f}$ represent the relaxation behaviour of the $\mathrm{H}$-bond among water molecules in the vicinity of each functional group. Comparing the $c_{\mathrm{ww}}(t)$ of two different DMA groups, the $c_{\mathrm{ww}}(t)$ of the unprotonated DMA groups, contrary to the $c_{\mathrm{pw}}(t)$, decays more slowly than that of the protonated DMA group at all temperature ranges. The mean relaxation time of the $\mathrm{H}$-bond among water molecules $\left(\tau_{\mathrm{ww}}\right)$ in the vicinity of the unprotonated DMA group is also slightly longer by about $12 \%$ than that of the protonated DMA group at 283 and $338 \mathrm{~K}$. It indicates that the unprotonated DMA group acts more as a hydrophobic solute by disturbing the H-bond exchange among water molecules, resulting in the longer $\tau_{\mathrm{ww}}$ in the vicinity of the unprotonated DMA group. ${ }^{61}$ The $c_{\mathrm{ww}}(t)$ of the carbonyl group surprisingly shows a decaying behaviour similar to that of the unprotonated
DMA group at $283 \mathrm{~K}$. Moreover, at higher temperatures (303 and $338 \mathrm{~K})$, the $c_{\mathrm{ww}}(t)$ decays even slower than that of the unprotonated DMA group, as shown in Fig. $7 \mathrm{~d}$ and $\mathrm{f}$. The $\tau_{\mathrm{ww}}$ in the vicinity of the carbonyl group is longer by about $18 \%$ and $26 \%$ than that of the protonated DMA group in the case of 303 and $338 \mathrm{~K}$, respectively. We observe that the $\mathrm{H}$-bond exchange among water molecules in the vicinity of the carbonyl group is mostly restricted in the globule state, with the longest $\mathrm{H}$-bond relaxation time of 0.68 ps. Although the carbonyl group maintains the $\mathrm{H}$-bond to water molecules, the extended $\tau_{\mathrm{ww}}$ in the vicinity of the carbonyl group can be associated with the polymer backbone near the carbonyl group, similar to the unprotonated DMA group.

\section{Residence time}

The motion of water molecules near the functional groups in PDMAEMA is also monitored to calculate the residence time, representing how long a water molecule will stay within a specific region. The residence time of water molecules in the vicinity of each functional group is calculated from the another TCF,

$$
r_{j}(t)=\left\langle p_{i}(t) p_{i}\left(t_{0}\right)\right\rangle_{j} /\left\langle p_{i}\left(t_{0}\right) p_{i}\left(t_{0}\right)\right\rangle_{j}
$$

where $p_{i}(t)=1$ if a water molecule $i$ is continuously in a particular region $j$ from time $t_{0}$ to $t$, and is zero otherwise. The regions $j$ for the unprotonated DMA group, the protonated DMA group, and the carbonyl group are set to be within $6.1 \AA$ of the $\mathrm{N}$, $\mathrm{Np}$, and $\mathrm{Oc}$ atom, respectively, which are identical to the regions for the $c_{\mathrm{ww}}(t)$. The angular brackets denote an average in a region. The residence time of water molecules in the particular region is calculated by integrating the TCF.

Fig. 8 displays the residence TCF of water molecules in the vicinity of each functional group. The residence time of water molecules decreases with increasing temperature due to the 
thermal energy. Compared with the unprotonated DMA group, the residence TCF of water molecules in the vicinity of the protonated DMA group, $r_{\mathrm{Np}}(t)$, decreases slowly, meaning that the water molecules stay longer around the protonated DMA group. However, there is no significant difference between the decreasing rate of $r_{\mathrm{N}}(t)$ and $r_{\mathrm{Np}}(t)$ with temperature change. In the case of the carbonyl group, we observe that the $r_{\mathrm{Oc}}(t)$ shows a slower decay than the $r_{\mathrm{Np}}(t)$ at 303 and $338 \mathrm{~K}$, while the $r_{\mathrm{Oc}}(t)$ shows a slightly faster decay than the $r_{\mathrm{Np}}(t)$ at $283 \mathrm{~K}$.

To understand this different tendency of the decay in the residence time for each functional group, we also calculated the residence time of water molecules in the first hydration shell, as shown in Table 3. It can be seen that the residence time of water molecules in the first hydration shell well correlates with its relaxation time of the H-bond between PDMAEMA and water, $\tau_{\mathrm{pw}}$. Since the motion of water molecules in the first hydration shell of each functional group is highly influenced by the
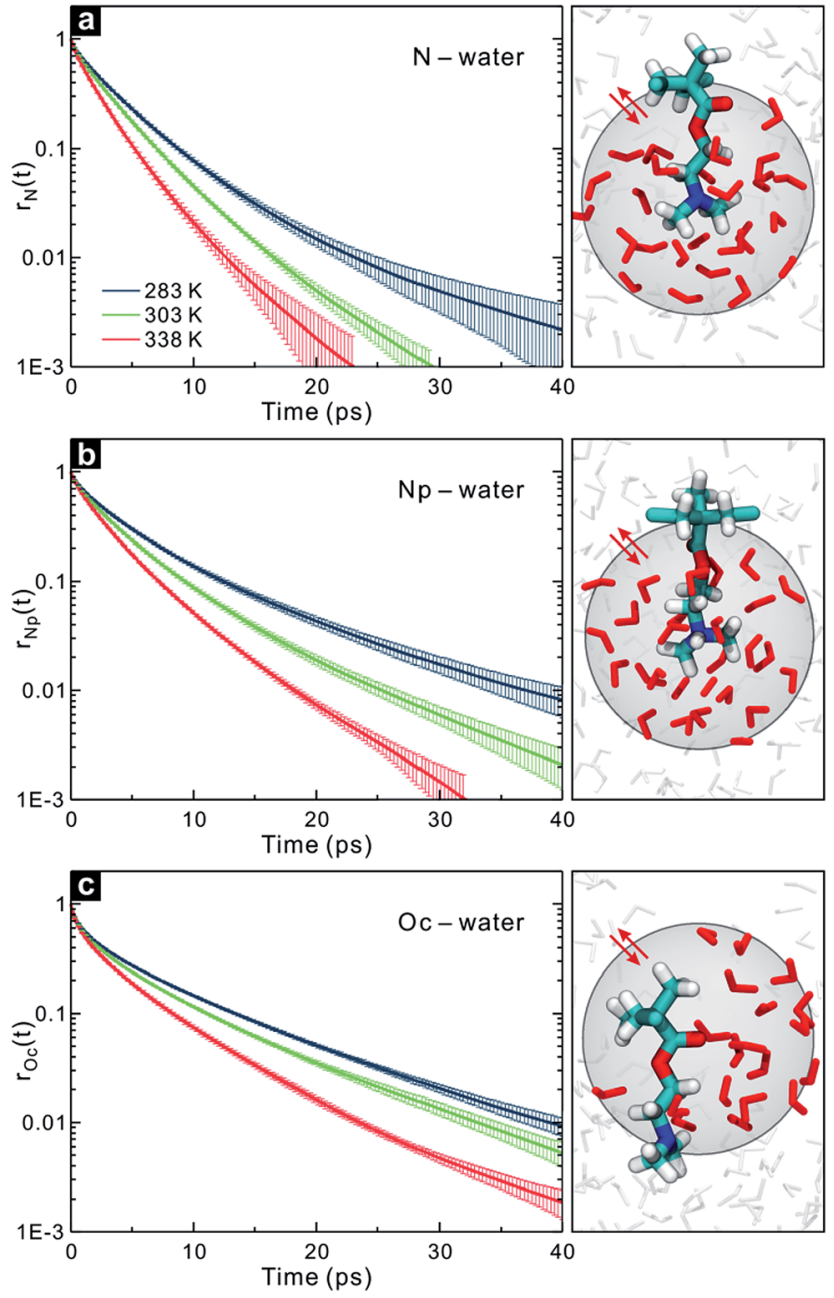

Fig. 8 Semi-log plots of residence TCF of water molecules in the vicinity of (a) the unprotonated DMA group, (b) the protonated DMA group, and (c) the carbonyl group after the production run at $T=283$, 303, and $338 \mathrm{~K}$. Snapshots represent the water molecules in the vicinity of each specific atom with the cutoff radius of $6.1 \AA$. For clarity, the water molecules in the inner and outer residence region are shown with red and grey rods, respectively. strength of $\mathrm{H}$-bonds between them, the residence time of the first hydration shell is obviously proportional to $\tau_{\mathrm{pw}}$. It is quite surprising to observe that the residence times in the vicinity of the carbonyl group at 303 and $338 \mathrm{~K}$ are 4.00 and $3.05 \mathrm{ps,}$ respectively, which are longer than those of the protonated DMA group, even though the residence time of water in the first hydration shell of the protonated DMA group is much longer. It is considered that this unusual residence time of water molecules in the vicinity of the carbonyl group is caused by the relatively low decreasing rate of the $\tau_{\mathrm{pw}}$ of the carbonyl group and by the extended $\tau_{\mathrm{ww}}$ in the vicinity of the carbonyl group.

From the H-bond relaxation time and the residence time of water molecules it may be concluded that the water molecules near the carbonyl group are restricted in motion with fewer opportunities for the exchange of $\mathrm{H}$-bonds. Although water molecules are also expelled from the unprotonated DMA groups which show the hydrophobic effect with the extended $\tau_{\mathrm{ww}}$ in the vicinity, the water dynamics surrounding the carbonyl group indicates a more restricted structure and temperature-sensitive behaviour compared with that surrounding the other functional groups, as the temperature increases. Thus, it can be suggested that the carbonyl group in PDMAEMA is more responsible for the coil-to-globule transition, involving the exclusion of water molecules with an entropic effect. This is consistent with the recent experimental study of Thavanesan et al. ${ }^{45}$ who reported that the change in solvation of the carbonyl group and backbone mainly leads to the phase transition of PDMAEMA.

\section{Protonation of the DMA group}

The degree of protonation was adjusted to investigate the effect of protonation on the conformational state of PDMAEMA where the DMA groups were fully protonated or unprotonated. According to the $\mathrm{p} K_{\mathrm{a}}$ value of PDMAEMA, the DMA groups almost appear in the form of protonation and deprotonation under acidic conditions and basic conditions, respectively. ${ }^{29}$ Fig. 9 shows the $R_{\mathrm{g}}$ of PDMAEMA with the fully protonated and unprotonated state of the DMA groups. The fully protonated PDMAEMA maintained the coil state through the production run at both 283 and even $338 \mathrm{~K}$ with a mean $R_{\mathrm{g}}$ of 16.8 and

Table 3 Residence time of water molecules in the first and up to second hydration shells of the unprotonated DMA, protonated DMA, and carbonyl group

\begin{tabular}{llll}
\hline & & \multicolumn{2}{l}{ Residence time $^{a}(\mathrm{ps})$} \\
\cline { 3 - 4 } Functional group & Temperature $(\mathrm{K})$ & First shell & Up to 6.1 \\
\hline Unprotonated DMA & 283 & $0.25 \pm 0.01$ & $3.56 \pm 0.12$ \\
& 303 & $0.22 \pm 0.01$ & $2.82 \pm 0.03$ \\
Protonated DMA & 338 & $0.18 \pm 0.00$ & $2.11 \pm 0.07$ \\
& 283 & $2.82 \pm 0.16$ & $4.95 \pm 0.20$ \\
Carbonyl & 303 & $2.37 \pm 0.20$ & $3.71 \pm 0.11$ \\
& 2838 & $1.55 \pm 0.05$ & $2.87 \pm 0.02$ \\
& 303 & $1.47 \pm 0.05$ & $4.78 \pm 0.10$ \\
& 338 & $1.32 \pm 0.09$ & $4.00 \pm 0.08$ \\
& & $1.05 \pm 0.01$ & $3.05 \pm 0.07$
\end{tabular}

${ }^{a}$ The residence time is calculated by integrating the residence TCF. 
16.9 $\AA$ for the last $10 \mathrm{~ns}$, respectively. On the other hand, from the initial equilibrium step, the fully unprotonated PDMAEMA chain collapsed into a globule state with a mean $R_{\mathrm{g}}$ of about $10 \AA$, even at $283 \mathrm{~K}$. It implies that PDMAEMA would be a watersoluble polyelectrolyte or becomes insoluble in water at the low and high $\mathrm{pH}$ conditions, respectively, and this is in good agreement with the experimental behaviour of PDMAEMA. ${ }^{29,43}$

The $R_{\mathrm{g}}$ values reveal that the fully protonated and unprotonated PDMAEMA prefer to be in the coil and globule state regardless of temperature changes, respectively. As expected, the N-Ow RDF for the fully unprotonated PDMAEMA shows a slight decrease in the intensity between the RDF peak at 283 and $338 \mathrm{~K}$, as shown in Fig. 10a, indicating that the unprotonated DMA group maintains its hydration state. Fig. 10b displays the Oc-Ow RDF of the fully unprotonated PDMAEMA. Interestingly, it can be seen that the intensity of the Oc-Ow RDF decreases significantly at $338 \mathrm{~K}$, with a decrease in $N_{\mathrm{w}}$ in the first hydration shell of Oc by $19 \%$. A similar phenomenon is found in the fully protonated PDMAEMA, as shown in Fig. 10c and d. The $N_{\mathrm{w}}$ in the first hydration shell of Oc is reduced by $16 \%$ in the fully protonated PDMAEMA at $338 \mathrm{~K}$, while that of $\mathrm{Np}$ is only reduced by $5 \%$.

Even though the fully unprotonated and protonated PDMAEMA maintain their conformational states, water molecules are still expelled from the vicinity of the carbonyl group with increasing temperature, irrespective of the protonation
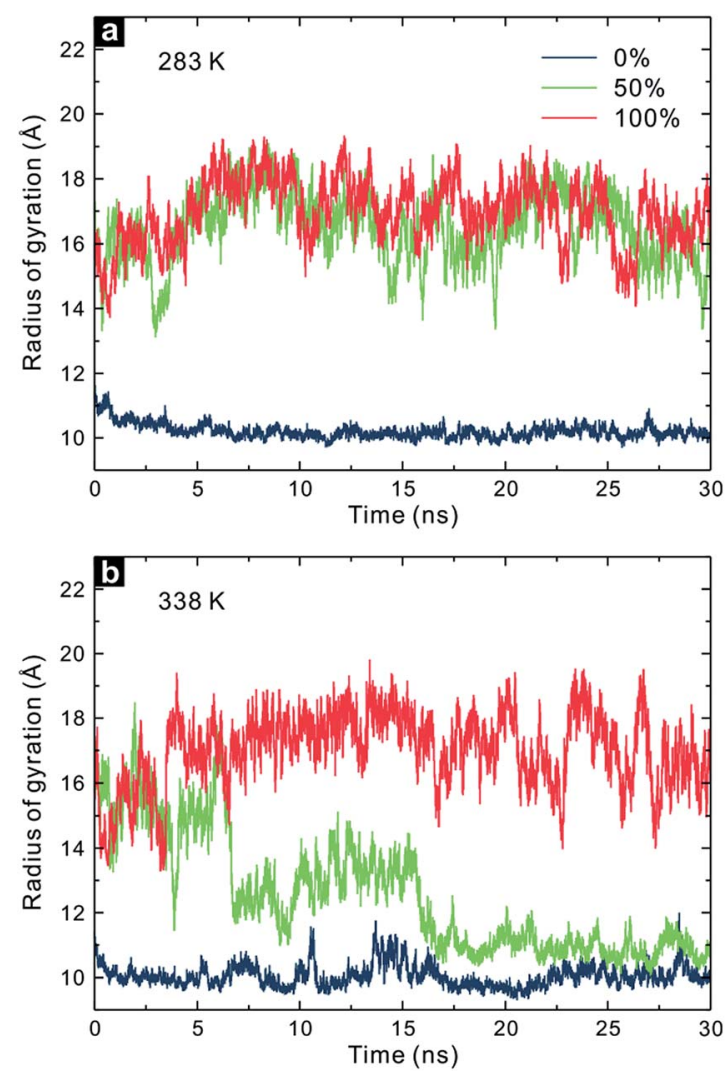

Fig. 9 Time evolution of the radius of gyration, $R_{\mathrm{g}}$, for PDMAEMA with the fully protonated (red), partially protonated (green), and unprotonated (blue) state at (a) $283 \mathrm{~K}$ and (b) $338 \mathrm{~K}$.
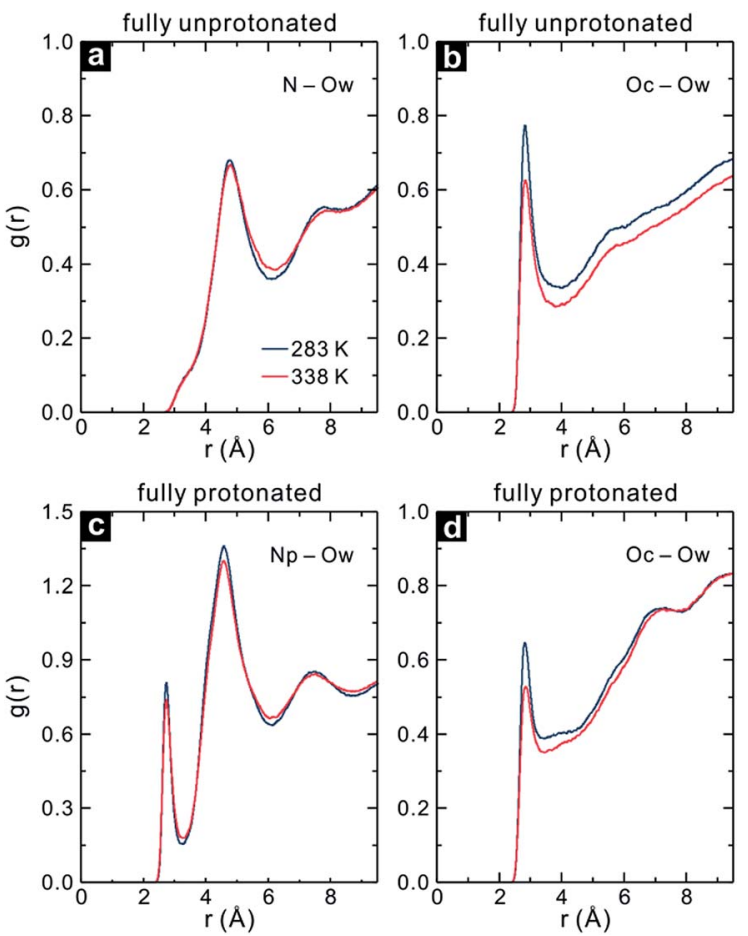

Fig. 10 RDFs of the (a) $\mathrm{N}$ and (b) Oc atom in the fully unprotonated PDMAEMA, and (c) $\mathrm{Np}$ and (d) Oc atom in the fully protonated PDMAEMA to water oxygen (OW) at 283 and $338 \mathrm{~K}$.

state of PDMAEMA. Thus, it is quite obvious that the carbonyl group plays a crucial role in the coil-to-globule transition in PDMAEMA. Moreover, it can be suggested that the DMA group can affect the solvation behaviour of PDMAEMA with the degree of its protonation in solution, resulting in the $\mathrm{pH}$-responsive property of PDMAEMA.

\section{Conclusions}

In this study, MD simulations have been successfully employed to observe the coil-to-globule transition of PDMAEMA in water and to understand the conformational state of PDMAEMA with atomic level at three different temperatures. The structural analysis, such as the $R_{\mathrm{g}}$ and WASA, as well as the pairwise energy analysis indicate that PDMAEMA shows a distinct conformational change through the LCST. The structural correlations between PDMAEMA and water were investigated to explore the hydration behaviour of the functional groups in the coil and globule state of PDMAEMA. From the RDFs of the functional groups in PDMAEMA it is observed that the protonated DMA group maintained its hydration state even in the globule state, while the unprotonated DMA group and the carbonyl group tended to lose their contact with water molecules. This expulsion of water molecules in the functional groups leads to the decrease in the number of $\mathrm{H}$-bonds between each functional group and water. In particular, it is found that the carbonyl group shows a significant decrease in the number of $\mathrm{H}$-bonds toward water molecules in the globule state. 
The motion of water molecules was further analysed to understand the conformational transition by monitoring the dynamic properties of water molecules in the vicinity of each functional group in detail. The residence time shows that the water molecules in the vicinity of the carbonyl group are more restricted in motion than other functional groups, with increasing temperature. This extended residence time results from the relatively low decreasing rate of the relaxation time of the H-bond between the carbonyl group and water, and from the extended relaxation time of the $\mathrm{H}$-bond among water molecules in the vicinity of the carbonyl group. Therefore, our simulations suggest that the motion of water molecules near the carbonyl group is restricted with fewer $\mathrm{H}$-bond exchange rates, which could be one of the possible driving forces for the coil-to-globule transition by the entropic effect.

The degree of protonation of the DMA group affects the solvation behaviour of PDMAEMA, resulting in its pH-responsiveness. Although the solvation behaviour of PDMAEMA depending on the degree of protonation is beyond the scope of this study, we note that the fully unprotonated and protonated PDMAEMAs maintain globule and coil states, respectively, regardless of temperature changes. The expulsion phenomenon of water molecules in the vicinity of the carbonyl group is also observed in the case of the fully unprotonated and protonated PDMAEMA, and it thus supports the role of each functional group in the conformational transition in PDMAEMA.

\section{Acknowledgements}

We thank the supercomputing resources of the UNIST Supercomputing Center. This work was supported by the National Research Foundation of Korea grant funded by the Korean Government (2010-0028684) and by the basic science research program (2013R1A1A2007491) through the National Research Foundation of Korea in the Ministry of Education, Science and Technology.

\section{Notes and references}

1 P. J. Flory, J. Chem. Phys., 1949, 17, 303-310.

2 F. Brochard and P. G. De Gennes, Macromolecules, 1977, 10, 1157-1161.

3 H. Feil, Y. H. Bae, J. Feijen and S. W. Kim, Macromolecules, 1993, 26, 2496-2500.

4 E. S. Gil and S. M. Hudson, Prog. Polym. Sci., 2004, 29, 11731222.

5 E. A. Clark and J. E. G. Lipson, Polymer, 2012, 53, 536-545.

6 T. M. Birshtein and V. A. Pryamitsyn, Macromolecules, 1991, 24, 1554-1560.

7 T. Hoare and D. McLean, Macromol. Theory Simul., 2006, 15, 619-632.

8 T. Koga, F. Tanaka, R. Motokawa, S. Koizumi and F. M. Winnik, Macromolecules, 2008, 41, 9413-9422.

9 Y. Tamai, H. Tanaka and K. Nakanishi, Macromolecules, 1996, 29, 6750-6760.

10 X. Wang, X. Qiu and C. Wu, Macromolecules, 1998, 31, 29722976.
11 Y. Zhang, S. Furyk, L. B. Sagle, Y. Cho, D. E. Bergbreiter and P. S. Cremer, J. Phys. Chem. C, 2007, 111, 8916-8924.

12 J.-F. Lutz, O. Akdemir and A. Hoth, J. Am. Chem. Soc., 2006, 128, 13046-13047.

13 M. Alaghemandi and E. Spohr, Macromol. Theory Simul., 2012, 21, 106-112.

14 M. Alaghemandi and E. Spohr, RSC Adv., 2013, 3, 3638-3647. 15 M. Alaghemandi and E. Spohr, Chem. Phys. Lett., 2013, 581, 80-84.

16 G. Longhi, F. Lebon, S. Abbate and S. L. Fornili, Chem. Phys. Lett., 2004, 386, 123-127.

17 H. Du, R. Wickramasinghe and X. Qian, J. Phys. Chem. B, 2010, 114, 16594-16604.

18 S. G. Lee, T. A. Pascal, W. Koh, G. F. Brunello, W. A. Goddard and S. S. Jang, J. Phys. Chem. C, 2012, 116, 15974-15985.

19 S. A. Deshmukh, S. K. R. S. Sankaranarayanan, K. Suthar and D. C. Mancini, J. Phys. Chem. B, 2012, 116, 2651-2663.

20 S. A. Deshmukh, S. K. R. S. Sankaranarayanan and D. C. Mancini, J. Phys. Chem. B, 2012, 116, 5501-5515.

21 S. A. Deshmukh, Z. Li, G. Kamath, K. J. Suthar, S. K. R. S. Sankaranarayanan and D. C. Mancini, Polymer, 2013, 54, 210-222.

22 X. Jiang, M. C. Lok and W. E. Hennink, Bioconjugate Chem., 2007, 18, 2077-2084.

23 C. V. Synatschke, A. Schallon, V. Jérôme, R. Freitag and A. H. E. Müller, Biomacromolecules, 2011, 12, 4247-4255.

24 C. Zhu, S. Jung, S. Luo, F. Meng, X. Zhu, T. G. Park and Z. Zhong, Biomaterials, 2010, 31, 2408-2416.

25 S. Agarwal, Y. Zhang, S. Maji and A. Greiner, Mater. Today, 2012, 15, 388-393.

26 H. Y. Cho, A. Srinivasan, J. Hong, E. Hsu, S. Liu, A. Shrivats, D. Kwak, A. K. Bohaty, H.-J. Paik, J. O. Hollinger and K. Matyjaszewski, Biomacromolecules, 2011, 12, 3478-3486.

27 J.-F. Gohy, S. Antoun and R. Jérôme, Macromolecules, 2001, 34, 7435-7440.

28 G. Liu, D. Wu, C. Ma, G. Zhang, H. Wang and S. Yang, ChemPhysChem, 2007, 8, 2254-2259.

29 H. Lee, S. H. Son, R. Sharma and Y.-Y. Won, J. Phys. Chem. B, 2011, 115, 844-860.

30 V. Bütün, S. Armes and N. Billingham, Polymer, 2001, 42, 5993-6008.

31 F. A. Plamper, A. Schmalz, M. Ballauff and A. H. E. Müller, J. Am. Chem. Soc., 2007, 129, 14538-14539.

32 X. Liu, P. Ni, J. He and M. Zhang, Macromolecules, 2010, 43, 4771-4781.

33 E. Betthausen, M. Drechsler, M. Förtsch, F. H. Schacher and A. H. E. Müller, Soft Matter, 2011, 7, 8880-8891.

34 H. Bao, L. Li, L. H. Gan, Y. Ping, J. Li and P. Ravi, Macromolecules, 2010, 43, 5679-5687.

35 X. Han, X. Zhang, H. Zhu, Q. Yin, H. Liu and Y. Hu, Langmuir, 2013, 29, 1024-1034.

36 F. A. Plamper, J. R. McKee, A. Laukkanen, A. Nykänen, A. Walther, J. Ruokolainen, V. Aseyev and H. Tenhu, Soft Matter, 2009, 5, 1812-1821.

37 S. B. Lee, A. J. Russell and K. Matyjaszewski, Biomacromolecules, 2003, 4, 1386-1393. 
38 X. J. Loh, S. J. Ong, Y. T. Tung and H. T. Choo, Polym. Chem., 2013, 4, 2564-2574.

39 W. Wu, J. Liu, S. Cao, H. Tan, J. Li, F. Xu and X. Zhang, Int. J. Pharm., 2011, 416, 104-109.

40 A. P. Majewski, A. Schallon, V. Jérôme, R. Freitag, A. H. E. Müller and H. Schmalz, Biomacromolecules, 2012, 13, 857-866.

41 Z. Dong, H. Wei, J. Mao, D. Wang, M. Yang, S. Bo and X. Ji, Polymer, 2012, 53, 2074-2084.

42 J. Niskanen, M. Karesoja, T. Rossi and H. Tenhu, Polym. Chem., 2011, 2, 2027-2036.

43 S. Dai, P. Ravi, C. H. Tan and K. C. Tam, Langmuir, 2004, 20, 8569-8575.

44 J. M. Bak, T. Lee, E. Seo, Y. Lee, H. M. Jeong, B.-S. Kim and H. Lee, Polymer, 2012, 53, 316-323.

45 T. Thavanesan, C. Herbert and F. A. Plamper, Langmuir, 2014, 30, 5609-5619.

46 H. Sun, J. Comput. Chem., 1994, 15, 752-768.

47 H. Sun, S. J. Mumby, J. R. Maple and A. T. Hagler, J. Am. Chem. Soc., 1994, 116, 2978-2987.

48 H. Sun, S. J. Mumby, J. R. Maple and A. T. Hagler, J. Phys. Chem., 1995, 99, 5873-5882.
49 S. Yang, S. Yu, W. Kyoung, D. Han and M. Cho, Polymer, 2012, 53, 623-633.

50 X. P. Chen, C. A. Yuan, C. K. Y. Wong, S. W. Koh and G. Q. Zhang, Mol. Simul., 2011, 37, 990-996.

51 A. K. Tucker and M. J. Stevens, Macromolecules, 2012, 45, 6697-6703.

52 B. Tangeysh, M. Fryd, M. A. Ilies and B. B. Wayland, Chem. Commun., 2012, 48, 8955-8957.

53 J. Niskanen, C. Wu, M. Ostrowski, G. G. Fuller, S. Hietala and H. Tenhu, Macromolecules, 2013, 46, 2331-2340.

54 S. Plimpton, J. Comput. Phys., 1995, 117, 1-19.

55 W. M. Brown, P. Wang, S. J. Plimpton and A. N. Tharrington, Comput. Phys. Commun., 2011, 182, 898-911.

56 F. S. Emami, V. Puddu, R. J. Berry, V. Varshney, S. V. Patwardhan, C. C. Perry and H. Heinz, Chem. Mater., 2014, 26, 2647-2658.

57 W. Li, J. Chem. Phys., 2013, 138, 014106.

58 W. Humphrey, A. Dalke and K. Schulten, J. Mol. Graphics, 1996, 14, 33-38.

59 A. Shrake and J. A. Rupley, J. Mol. Biol., 1973, 79, 351-371. 60 A. Luzar, J. Chem. Phys., 2000, 113, 10663-10675.

61 H. Xu and B. J. Berne, J. Phys. Chem. B, 2001, 105, 1192911932. 